\title{
Prevalence and Vulnerability to Drug Abuse among Students of High School and Colleges in Riyadh, Saudi Arabia: Cross-Sectional Study by Khadeejeh, Alaa, Nahla, Nourah, Alaa, Beshaier, and Hind (2019)
}

\author{
Alqahtani Jawhrah Mohammed \\ King Saud University, School of Nursing, Riyadh 11451, Saudi Arabia, \\ Email: jawhrah.alqahtani@mymail.barry.edu.
}

Dear Editor,

I am writing in response to the February 2019 article titled "Prevalence and Vulnerability to Drug Abuse among Students of High School and Colleges in Riyadh, Saudi Arabia: CrossSectional Study." I believe you have identified a serious threat to teenagers of both genders and the community as a whole. Substance abuse is a globally well-recognized issue and is defined as a chronic "relapsing" disease, affecting all aspects of indivduals' lives. ${ }^{[2]}$ In today's society, these populations oftentimes experience a lot of stressful situations and challenges regarding self-identity, sexuality, drugs, schoolwork, and social life. Being in the transition stage to maturity can produce confusion and poor judgment, so they are at high risk for consuming drugs. ${ }^{[2]}$

\section{Discussion}

Since adolescents spend the largest portion of their time at school, the school is the ideal location for identification of teenagers at risk for substance abuse and meeting their needs as early as possible. ${ }^{[1]}$ The American Academy of Pediatrics Council on School Health (2016) acknowledges the essential role that school nurses have in the student's continuum of holistic care and academic success. School nurses are in a position to reach a large number of adolescents, recognize their problem, and foster their general wellbeing and educational success. Through a daily presence, the school nurse can contribute to adolescent health positively and to the reduction of adolescent addiction. ${ }^{[3]}$ Evidence shows that early identification of addiction has a significant impact on patient outcomes $;^{[2]}$ thus, having a protocol to screen teenagers by a school nurse periodically for early signs and symptoms of drug addiction is beneficial.

In the school setting, the school administrators and teachers are also in a key position to monitor the adolescents' behaviors closely, help them to deal with stressors, and prevent further academic and behavior disparities. ${ }^{[1]}$ Social support and affirming the value of the patient's voice help establish a platform for a meaningful relationship where anything can be shared and solved. ${ }^{[3]}$ Students' awareness of the role of the school nurse and teachers can aid in communication and assessment of needs. Interprofessional collaboration among school professionals is significant to assess the needs of students, enhance students' achievement, and ensure the coordinated care of students while in school. ${ }^{[3]}$

\section{Conclusion}

Although this issue has become one of the most dominant among adolescents in communities across the nation, a universal lack of knowledge about addiction is glaring, especially among the parents. Educating adolescents, parents, and society on the seriousness of drug addiction, its contributory factors, and how to deal with it on local and global levels, are needed to enhance the general well-being of adolescents to be more independent and productive citizens. By having more awareness, both families and students are better able to recognize the concept of drug addiction and engage in treatment and prevention.

\section{References}

[1] American Academy of Pediatrics, Council on School Health. (2016). Role of the school nurse in providing school health services. Pediatrics, 137(6), e20160852. https://pediatrics.aappublications.org/content/pediatrics/e arly/2016/05/19/peds.2016-0852.full.pdf

[2] Centers for Disease Control and Prevention. (2014). Whole school, whole community, whole child, a collaborative approach to learning and health. https://www.cdc.gov/healthyyouth/wscc/

[3] National Association of School Nurses. (2015). Framework for 21st century school nursing practice. NASN School Nurse, 130(4), 219-231. https://journals.sagepub.com/doi/10.1177/1942602X155 89559

[4] Smolowitz, J., Speakman, E., \& Wojner, D. (2014). Role of the registered nurse in primary health care: Meeting health care needs in the 21 st century. Science Direct, http://dx.doi.org/10.1016/j.outlook.2014.08.004 


\section{Author Profile}

Alqahtani Jawhrah received a B.S. degree in Nursing from King Saud University, and received an M.S degree in Nursing Education from Roberts Wesleyan College in the United States of America respectively. Currently, she is completing her $\mathrm{PhD}$ in Nursing at Barry University in the state of Florida, the United States of America. 